\title{
Incidence, risk factors and clinical significance of postoperative haemodynamic instability after adrenalectomy for phaeochromocytoma
}

\author{
Joseph P. Thompson ${ }^{1}$, Davinia Bennett ${ }^{2}$, James Hodson ${ }^{3}$, Miriam Asia $^{4,5}$, John Ayuk ${ }^{4,5}$, Michael W. \\ O'Reilly $^{4,5,6}$, Niki Karavitaki ${ }^{4,5,6}$, Wiebke Arltt ${ }^{4,5,6}$, Robert P. Sutcliffe ${ }^{1}$
}

${ }^{1}$ HPB Surgery Unit, ${ }^{2}$ Anaesthetic Department, ${ }^{3}$ Department of Biostatistics, Institute of Translational Medicine, ${ }^{4}$ Department of Endocrinology, Queen Elizabeth Hospital Birmingham, Birmingham, UK; ${ }^{5}$ Institute of Metabolism and Systems Research, College of Medical and Dental Sciences, University of Birmingham, Birmingham, UK; ${ }^{6}$ Centre for Endocrinology, Diabetes and Metabolism, Birmingham Health Partners, Birmingham, UK Contributions: (I) Conception and design: RP Sutcliffe, D Bennett, J Ayuk, J Hodson, MW O'Reilly, N Karavitaki, W Arlt, M Asia; (II) Administrative support: None; (III) Provision of study materials or patients: RP Sutcliffe, J Ayuk; (IV) Collection and assembly of data: JP Thompson, RP Sutcliffe, D Bennett; (V) Data analysis and interpretation: RP Sutcliffe, J Hodson, JP Thompson, D Bennett; (VI) Manuscript writing: All authors; (VII) Final approval of manuscript: All authors.

Correspondence to: Robert P. Sutcliffe. HPB Surgery Unit, Third floor Nuffield House, Queen Elizabeth Hospital, Birmingham B15 2TH, UK. Email: robert.sutcliffe@uhb.nhs.uk.

Background: Due to risk of haemodynamic instability (HDI), it has been recommended that patients undergoing adrenalectomy for phaeochromocytoma should be monitored in an intensive care facility. The aim of this study was to evaluate the incidence, risk factors and outcomes of postoperative HDI in these patients. Retrospective cohort study of 46 consecutive patients who underwent open (OA, N=26) or laparoscopic (LA, N=20) adrenalectomy for phaeochromocytoma at a single centre [2007-2017].

Methods: HDI was defined as systolic BP $>200$ or $<90 \mathrm{mmHg}$, heart rate $>120$ or $<50 \mathrm{bpm}$ or vasopressor therapy within 24 hours. Risk factors for intraoperative and postoperative HDI were evaluated by univariable and multivariable analyses.

Results: Intraoperative hypertension occurred in 25/42 patients (60\%). Preoperative plasma normetanephrine levels $\geq 3,500 \mathrm{pmol} / \mathrm{L}$ were significantly associated with intraoperative hypertension on multivariable analysis [odds ratio (OR) 42; 95\% CI: 4-429; $\mathrm{P}=0.002)$. Postoperative hypotension occurred in $21 / 45$ patients (47\%), and 13 (29\%) required vasopressor therapy. Preoperative beta-blockade therapy was the only independent risk factor for postoperative hypotension on multivariable analysis (OR 4.0; $95 \%$ CI: $1.2-13.9, \mathrm{P}=0.029)$. No patients (0/9) with tumours $<5 \mathrm{~cm}$ treated by LA needed postoperative vasopressor therapy, compared to $39 \%(7 / 18)$ treated by OA $(\mathrm{P}=0.059)$. Complications developed in 9 patients $(20 \%)$, and were less likely in those with intraoperative hypertension $(8 \%$ vs. $41 \% ; \mathrm{P}=0.019)$. There was one postoperative death.

Conclusions: Preoperative beta-blockade therapy is an independent risk factor for postoperative HDI after adrenalectomy for phaeochromocytoma. Patients who undergo laparoscopic adrenalectomy (LA) for phaeochromocytomas $<5 \mathrm{~cm}$ are unlikely to need postoperative vasopressor therapy, and may not require intensive care monitoring.

Keywords: Phaeochromocytoma; adrenalectomy; haemodynamic instability (HDI)

Submitted Aug 06, 2019. Accepted for publication Nov 04, 2019.

doi: $10.21037 /$ gs.2019.11.22

View this article at: http://dx.doi.org/10.21037/gs.2019.11.22 


\section{Introduction}

Phaeochromocytomas are rare catecholamine producing tumours arising from the chromaffin cells of the adrenal medulla (1). Excess circulating catecholamines are responsible for the clinical manifestations of the tumour, and include palpitations, hypertension and potentially lifethreatening complications, such as myocardial infarction or stroke (2). Surgical resection is the only curative treatment, but was historically associated with a significant mortality risk, primarily due to intraoperative haemodynamic instability (HDI) (3-6). In recent decades, the mortality risk from adrenalectomy for phaeochromocytoma has reduced significantly, due to advances in both preoperative and perioperative management (7-10). Nevertheless, HDI remains a common occurrence, particularly during endotracheal intubation, creation of pneumoperitoneum (during laparoscopic surgery) and after tumour manipulation (3,5,11-13) and is associated with increased postoperative morbidity (14). Frequent peaks of systolic hypertension necessitate prompt intervention with vasoactive agents, and may be followed by episodes of hypotension after adrenal vein ligation during surgery. Given that HDI, particularly rebound hypotension, may also occur postoperatively, it has been recommended that all patients should be monitored in a high dependency or intensive care environment (15). The risk factors for intraoperative HDI have been evaluated in several studies, and include tumour size, catecholamine levels, preoperative mean arterial blood pressure and operative approach $(9,16)$. By contrast, the incidence and risk factors for postoperative HDI have not been well defined. Benay et al. found that catecholamine levels, preoperative mean arterial blood pressure and resection of an adjacent organ were significantly associated with postoperative HDI, and they proposed a selective approach to use of intensive care monitoring in high risk patients (17). The aim of this study was to determine the incidence, risk factors and outcome of postoperative HDI after adrenalectomy for phaeochromocytoma in a series of consecutive patients from a tertiary UK referral centre.

\section{Methods}

This was a retrospective study using data collected from a prospectively maintained institutional database. The study was registered and approved as a clinical audit by University of Birmingham NHS Foundation Trust (Audit number CARMS-13194). Informed consent was waived in accordance with the ethical committee opinion.

\section{Preoperative work-up}

The diagnosis of phaeochromocytoma was made preoperatively by detection of elevated levels of either 24-hour urinary or plasma catecholamine levels in combination with the presence of an adrenal mass on crosssectional imaging (CT or MRI scan). In our Unit, plasma metanephrine measurement has superseded 24-hour urine collection as the preferred method of detection since 2012 . After diagnosis, all patients underwent an iodine-123 metaiodobenzylguanidine (MIBG) scan (to localise tumours and exclude metastatic disease) and were commenced on alpha-adrenergic blockade with or without beta-adrenergic blockade. The choice of adrenergic blockade has evolved during the study period: between 2007 and 2012, patients were routinely alpha- and beta-blocked with a combination of Phenoxybenzamine (or Doxazosin) and Propranolol (or Bisoprolol). Beta-blockade was used more selectively from 2013 in patients who developed tachycardia after alphablockade, and Doxazosin became the preferred alphablocker since 2014. All patients were admitted the evening before surgery and received an intravenous fluid infusion to prevent hypovolaemia prior to induction of anaesthesia.

\section{Surgical technique}

Between 2007 and 2014, all patients underwent adrenalectomy by an open approach using a subcostal incision. A thoracic epidural catheter was employed for perioperative analgesia according to anaesthetist and patient preference. Since June 2014, all adrenalectomies for benign tumours (including phaeochromocytoma) and small malignant tumours have been performed laparoscopically. For laparoscopic cases, a transperitoneal approach has been employed, with patients in a lateral decubitus position. During both open and laparoscopic procedures, tumour handling was avoided until adrenal vein ligation.

\section{Anaesthetic technique}

All patients were managed by an experienced anaesthetist. Propofol was used for induction in all cases, and anaesthesia maintained with volatile agents. Remifentanil was used for perioperative analgesia and to aid blood pressure control. Haemodynamic parameters (central venous pressure, blood pressure) were continuously monitored by central venous 
and arterial catheters. Intraoperative hypertension was treated by increased depth of anaesthesia (Remifentanil) and vasodilator agents (alone or in combination) according to anaesthetist's preference (e.g., Sodium Nitroprusside, Glyceryl Trinitrate, Labetalol, Phentolamine, Esmolol, Magnesium). After adrenal vein ligation, intraoperative hypotension was treated by intravenous fluid therapy and/or vasopressor agents (Noradrenaline, Metaraminol, Ephedrine). Postoperatively, all patients were monitored in a high dependency unit. Postoperative hypotension was treated with vasopressor agents (Noradrenaline) after excluding hypovolaemia.

\section{Data collection}

All patients who underwent adrenalectomy between January 2007 and December 2017 were identified from a prospective institutional database. Data were collected on patient demographics, plasma/urinary catecholamine levels and preoperative antihypertensive therapy (drug and dose). Intraoperative and postoperative data regarding haemodynamic variables were obtained by review of anaesthetic charts and electronic patient records. The following definitions were used: (I) intraoperative hypertension $=$ systolic blood pressure $>200 \mathrm{mmHg}$ and/or need for vasodilator therapy; (II) intraoperative/ postoperative hypotension = systolic blood pressure $<90 \mathrm{mmHg}$ and/or need for vasopressor therapy; (III) sinus tachycardia $=$ heart rate $>120$ beats per minute $(\mathrm{bpm})$; and (IV) sinus bradycardia $=$ heart rate $<50 \mathrm{bpm}$. The volume of intravenous fluid administered during and after surgery was recorded for each patient. The presence and severity of postoperative complications (according to the ClavienDindo classification) (18) and length of hospital stay were also recorded. This study was performed retrospectively and did not involve any interventions beyond standard care.

\section{Statistical analysis}

Normally distributed variables were reported as means \pm standard deviations (SDs) and analysed using independent samples $t$-tests, whilst ordinal and non-normal variables were reported as medians and interquartile ranges (IQRs) and analysed using Mann-Whitney tests. Binary factors were compared using Fisher's exact tests. Multivariable analyses were then performed, in an attempt to identify independent predictors of hypo- and hypertension. Binary logistic regression models were produced, using a forward stepwise approach to variable selection. For intraoperative outcomes, all preoperative factors were considered for inclusion. For postoperative outcomes, intraoperative fluid volume and hypertension were additionally considered, whilst analysis of length of stay additionally considered the postoperative factors for inclusion. Continuous variables were dichotomised based on the median value, as reliable goodness of fit testing was not possible in light of the small sample size. Factors with large quantities of missing data that were not selected for inclusion in the final model were then excluded, and the analysis repeated, to maximise the included sample size. All analyses were performed using IBM SPSS 22 (IBM Corp. Armonk, NY, USA), with $\mathrm{P}<0.05$ deemed to be indicative of statistical significance throughout.

\section{Results}

\section{Patient demographics}

During the study period, 46 consecutive patients underwent either unilateral $(\mathrm{N}=42)$ or bilateral $(\mathrm{N}=4)$ adrenalectomy for phaeochromocytoma. The mean age at surgery was $51 \pm 16$ years and $74 \%(\mathrm{~N}=34)$ were female. No patients had evidence of metastatic disease. Thirty-nine of 46 patients were diagnosed with phaeochromocytoma on the basis of elevated plasma and/or urinary metanephrines preoperatively; one patient underwent surgery in 2007 for an incidental adrenal tumor without prior biochemical assessment for phaeochromocytoma and in six patients, preoperative plasma metanephrine levels were within the normal range. However, in all 46 patients a diagnosis of phaeochromocytoma was confirmed by postoperative histopathology. Where available $(\mathrm{N}=37)$, the median plasma normetanephrine level was $3,138 \mathrm{pmol} / \mathrm{L}$ (range, $40-25,000)$ [reference range $<1,180$ ]. The median tumour size was $4.5 \mathrm{~cm}$ (range, 1.1-13.0) and eleven patients (24\%) had an underlying genetic syndrome: neurofibromatosis type $1(\mathrm{~N}=5)$, multiple endocrine neoplasia type $2(\mathrm{~N}=3)$ and von Hippel Lindau syndrome $(\mathrm{N}=3)$. Preoperatively, 20 patients received alpha-blockade only (43\%) and 21 patients received both alpha and beta-blockade (47\%). Five patients did not receive preoperative alpha-blockade, as the diagnosis of phaeochromocytoma was not considered, either due to normal or minor elevation of plasma normetanephrines $(\mathrm{N}=4)$ or lack of available preoperative catecholamine value $(\mathrm{N}=1)$ (see above). Two of these patients were taking regular beta-blockers for treatment 
Table 1 Phaeochromocytoma patient demographics and outcomes—open vs. laparoscopic adrenalectomy

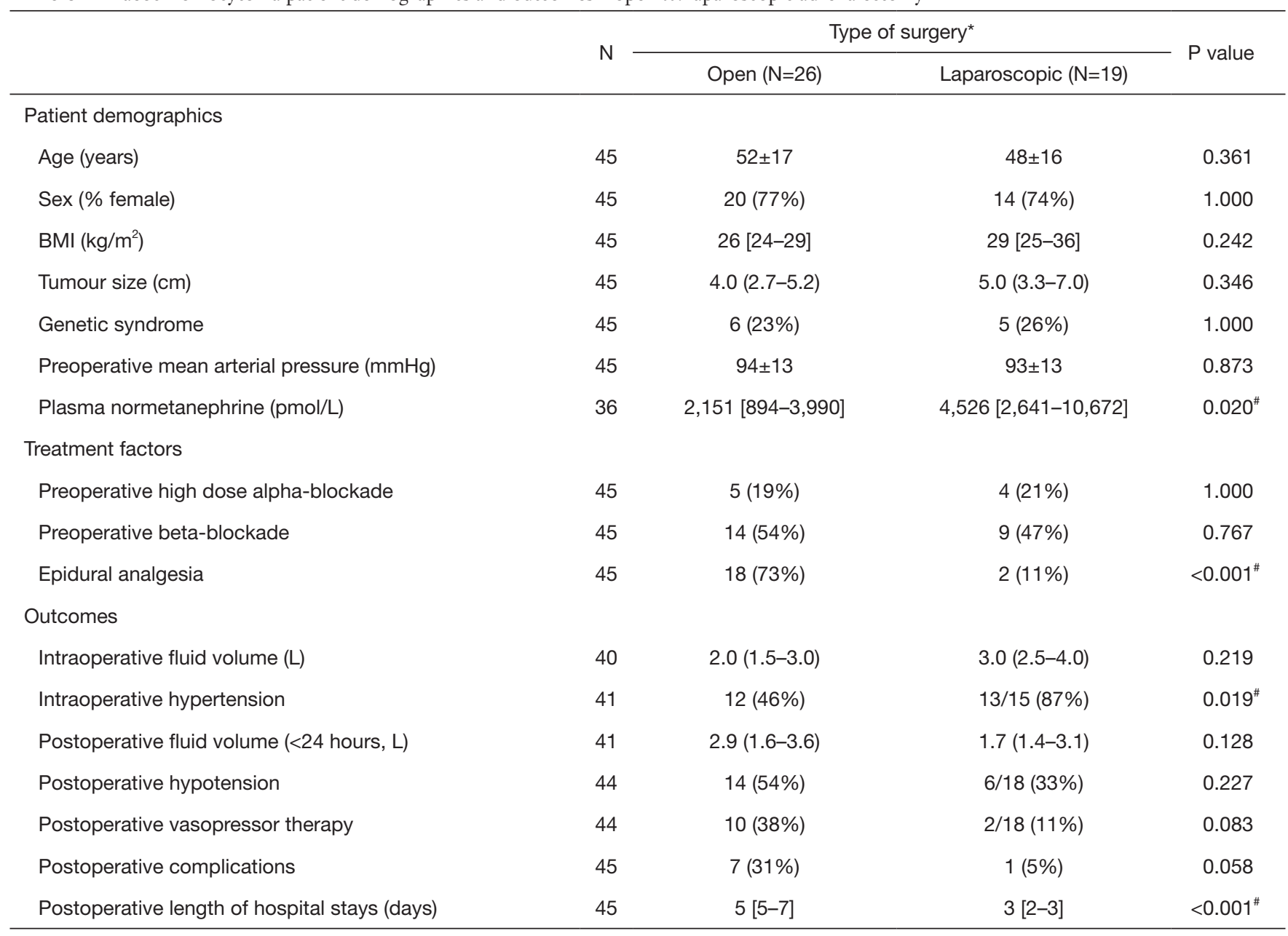

Binary factors are reported as $\mathrm{n}(\%)$ or as $\mathrm{n} / \mathrm{N}(\%)$ where there are missing data, with $\mathrm{P}$ values from Fisher's exact tests. Continuous factors are reported as mean $\pm \mathrm{SD}$, with $\mathrm{P}$ values from $t$-tests, or as median (IQR), with $\mathrm{P}$ values from Mann-Whitney tests. " $\mathrm{P}<0.05$. *, excludes one patient whose laparoscopic procedure was converted to open surgery.

of presumed essential hypertension. The 41 patients who were alpha-blocked were treated with doxazosin $(\mathrm{N}=29)$, phenoxybenzamine $(\mathrm{N}=11)$ or prazosin $(\mathrm{N}=1)$. Of those patients who were treated with doxazosin, 13 (45\%) received a daily dose $<8 \mathrm{mg}$ and $16(55 \%)$ received a daily dose $\geq 8 \mathrm{mg}$. A total of 23 patients (50\%) received preoperative beta-blockade, either non-selective ( $\mathrm{N}=11$, propranolol) or beta-1-selective ( $\mathrm{N}=12$; atenolol, bisoprolol).

\section{Surgical approach}

Overall, 26 patients (57\%) underwent open adrenalectomy (OA), including the first 25 consecutive patients in the series (June 2007-May 2014). Since the laparoscopic programme began in June 2014, 20 of 21 patients (95\%) have undergone laparoscopic adrenalectomy (LA), with the remaining patient undergoing open surgery due to tumour size $(13 \mathrm{~cm})$. In addition, one patient in the LA group $(5 \%)$ underwent conversion to open surgery due to involvement of the renal vein, which was resected and reconstructed. This patient was excluded from analysis of surgical approach, such that groups being compared were $\mathrm{OA}(\mathrm{N}=26)$ and LA $(\mathrm{N}=19)$. Comparisons between these two groups (Table 1) found no significant differences in patient demographics, although plasma normetanephrine levels were significantly higher in the LA group (median: $4,526$ vs. 2,151; $\mathrm{P}=0.020)$. Of the treatment-related factors, the rates of preoperative high dose alpha-blockade 
Table 2 Factors associated with intraoperative hypertension

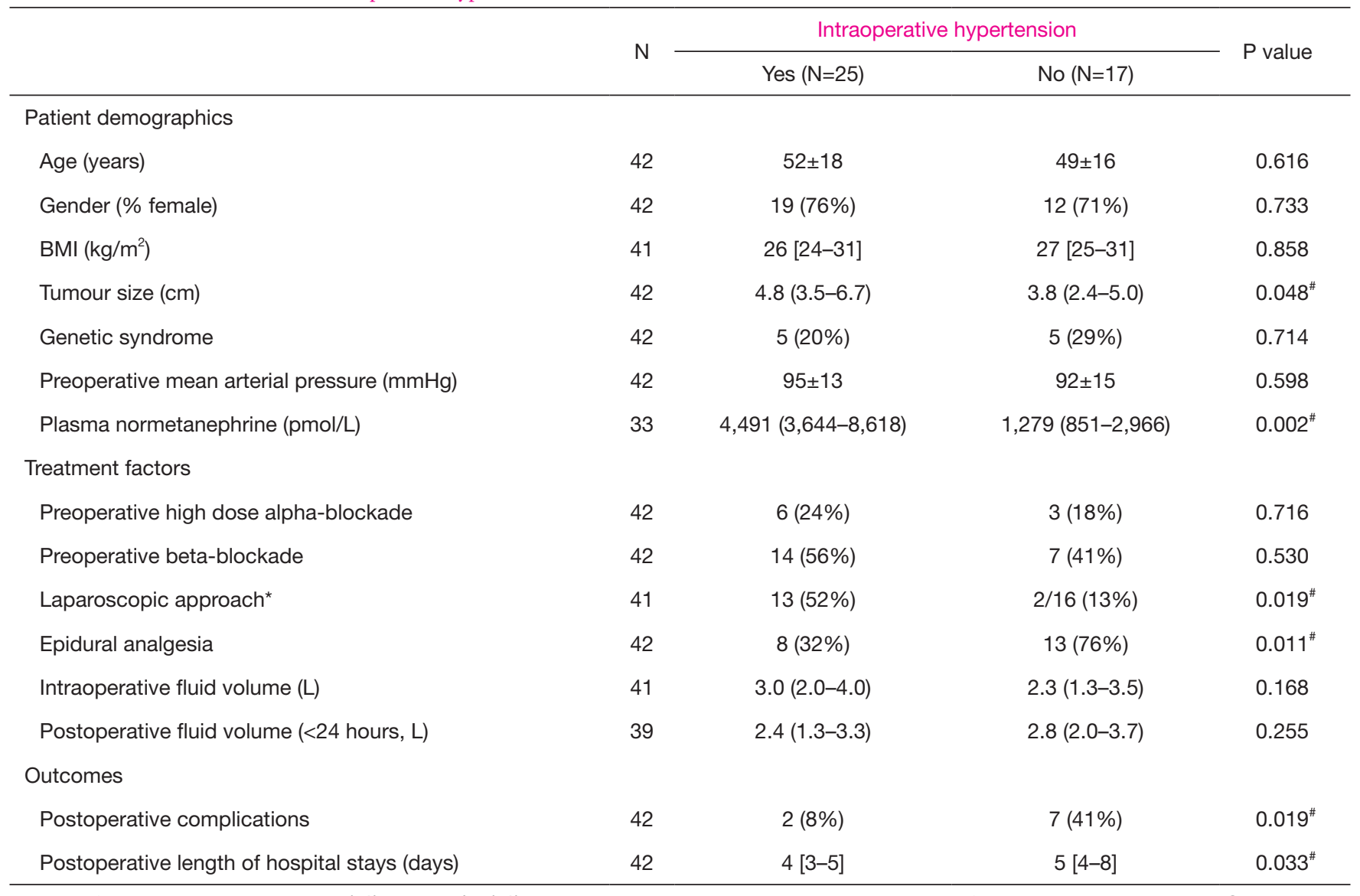

Binary factors are reported as $\mathrm{n}(\%)$ or as $\mathrm{n} / \mathrm{N}(\%)$ where there are missing data, with $\mathrm{P}$ values from Fisher's exact tests. Continuous factors are reported as mean $\pm \mathrm{SD}$, with $\mathrm{P}$ values from $t$-tests, or as median (IQR), with $\mathrm{P}$ values from Mann-Whitney tests. ", $\mathrm{P}<0.05$. *, excludes one patient whose laparoscopic procedure was converted to open surgery.

$(\mathrm{P}=1.000)$ and beta-blockade $(\mathrm{P}=0.767)$ usage were similar between groups. However, the use of epidural analgesia was significantly more common in OA $(73 \%)$ compared to LA $(11 \% ; \mathrm{P}<0.001)$.

\section{Intraoperative HDI}

Intraoperative haemodynamic data was available for 42 patients $(91 \%)$. Of these, 25 patients $(60 \%)$ had a median of 3.5 episodes (range, 1-30) of intraoperative systolic hypertension $(>200 \mathrm{mmHg})$, including 22 patients who required vasodilator therapy. A single agent was used in 18 patients (Esmolol, Glycerol Trinitrate, Labetalol, Phentolamine, Sodium Nitroprusside), and multiple agents were used in four patients. Thirty-seven patients (88\%) had a median of 3 episodes (range, 1-21) of intraoperative hypotension $(<90 \mathrm{mmHg}$ ), including 29 patients who required vasopressor therapy (Metaraminol, Noradrenaline, Ephedrine).

Intraoperative hypertension was significantly more common during LA compared to OA, with rates of $87 \% v s$. $46 \%$, respectively $(\mathrm{P}=0.019)$. However, patients operated by LA had significantly higher plasma normetanephrine levels and were less likely to have epidural analgesia (11\% vs. $73 \%, \mathrm{P}<0.001$ ) (Table 1). Both of these factors were also found to be significantly associated with intraoperative hypertension on univariable analysis (Table 2). Indeed, on multivariable analysis, only the plasma normetanephrine level was found to be a significant independent predictor of intraoperative hypertension, with an odds ratio of 42 (95\% CI: 4-429, P=0.002) for levels of greater than $3,500 \mathrm{pmol} / \mathrm{L}$.

A total of 10 patients (24\%) had a median of 4 episodes (range, 1-24) of sinus tachycardia ( $>120 \mathrm{bpm})$, whilst 10 patients (24\%) had a median of 3 episodes (range, 1-8) 
Table 3 Factors associated with postoperative hypotension

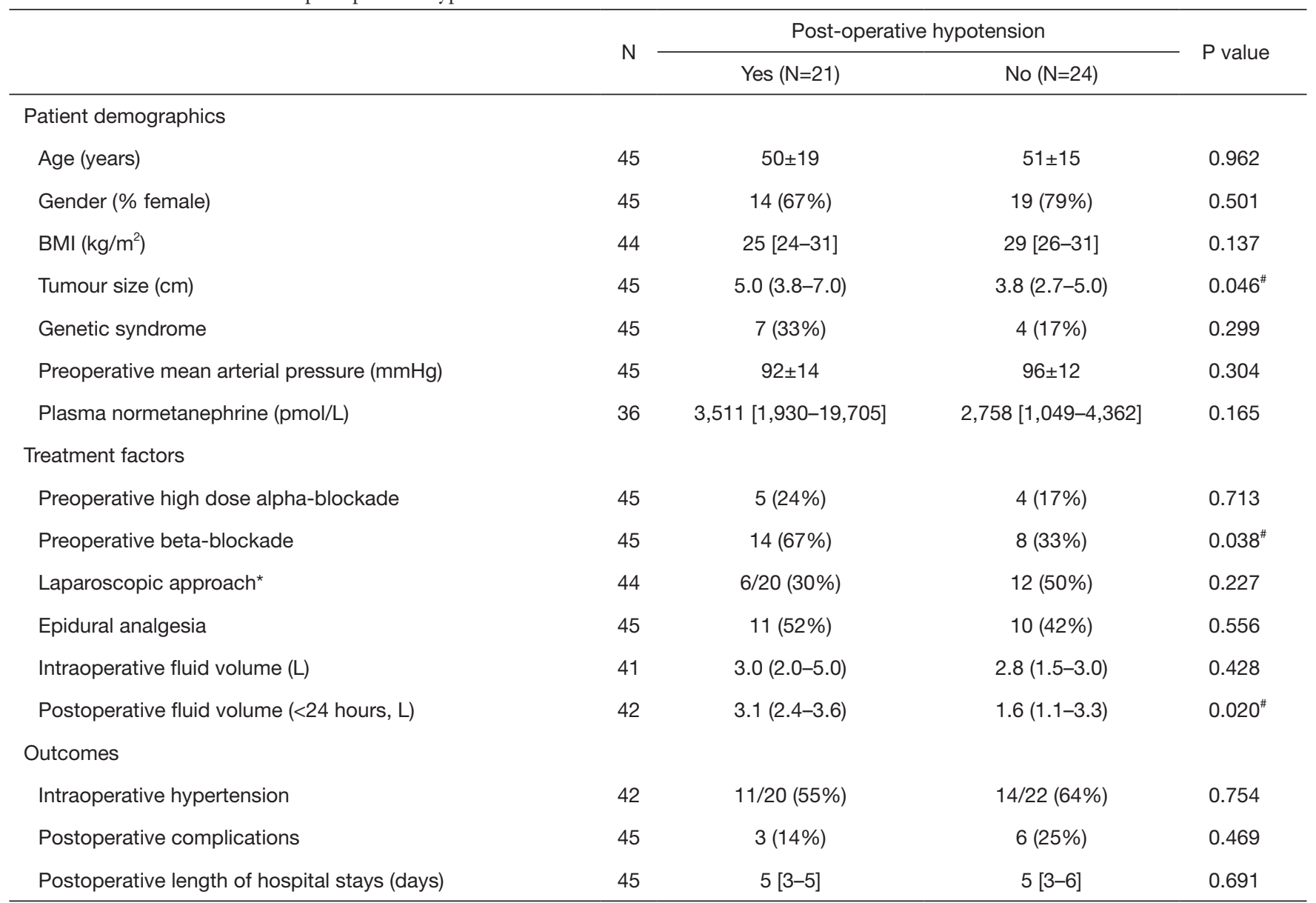

Binary factors are reported as $\mathrm{n}(\%)$ or as $\mathrm{n} / \mathrm{N}(\%)$ where there are missing data, with $\mathrm{P}$ values from Fisher's exact tests. Continuous factors are reported as mean $\pm \mathrm{SD}$, with $\mathrm{P}$ values from $t$-tests, or as median (IQR), with $\mathrm{P}$ values from Mann-Whitney tests. " $\mathrm{P}<0.05$; * excludes one patient whose laparoscopic procedure was converted to open surgery.

of sinus bradycardia (<50 bpm). The median volume of intravenous fluid administered intraoperatively was 3.0 litres (range, 0.5-7.0) and one patient required an intraoperative blood transfusion.

\section{Postoperative HDI}

Postoperative haemodynamic data was available for 45 patients (98\%); 26 patients $(58 \%)$ had evidence of postoperative HDI. A total of 21 patients (47\%) had postoperative hypotension (<90 $\mathrm{mmHg}$ ), including 13 (29\%) who required vasopressor therapy within the first 24 hours. Postoperatively, four patients had a median of 2.5 episodes (range, 1-6) of sinus bradycardia $(<50 \mathrm{bpm})$ and one patient had an episode of sinus tachycardia. Patients with postoperative hypotension had significantly larger tumours (median: 5.0 vs. $3.8 \mathrm{~cm}, \mathrm{P}=0.046$ ) and were significantly more likely to be beta-blocked preoperatively (67\% vs. $33 \%, \mathrm{P}=0.038$, Table 3). On multivariable analysis, only the latter was found to be a significant independent predictor of postoperative hypotension, with an odds ratio of 4.0 (95\% CI: $1.2-13.9, \mathrm{P}=0.029)$ for those patients on preoperative beta-blockers compared to those that were not.

None of the pre- or intraoperative factors considered were found to be significantly predictive of the need for postoperative vasopressor therapy (Table 4). However, there was a tendency for postoperative vasopressor therapy to be less common after laparoscopic compared to open surgery ( $11 \%$ vs. $38 \% ; \mathrm{P}=0.083)$. No patients with tumours less than $5 \mathrm{~cm}$ treated by LA (0/9) required postoperative vasopressor therapy compared to $39 \%(7 / 18)$ in the OA group $(\mathrm{P}=0.059)$. The volume of intravenous fluid administered within the 
Table 4 Factors associated with postoperative vasopressor therapy

\begin{tabular}{|c|c|c|c|c|}
\hline & $\mathrm{N}$ & \multicolumn{2}{|c|}{ Postoperative vasopressor therapy } & $P$ value \\
\hline \multicolumn{5}{|l|}{ Patient demographics } \\
\hline Age (years) & 45 & $54 \pm 21$ & $49 \pm 15$ & 0.391 \\
\hline Gender (\% female) & 45 & $8(62 \%)$ & $25(78 \%)$ & 0.285 \\
\hline Tumour size $(\mathrm{cm})$ & 45 & $4.8(4.1-5.2)$ & $4.0(2.9-5.1)$ & 0.239 \\
\hline Genetic syndrome & 45 & $3(23 \%)$ & $8(25 \%)$ & 1.000 \\
\hline Preoperative mean arterial pressure $(\mathrm{mmHg})$ & 45 & $91 \pm 16$ & $95 \pm 12$ & 0.307 \\
\hline Plasma normetanephrine (pmol/L) & 36 & $2,966[1,219-8,618]$ & $3,644[1,246-5,063]$ & 0.992 \\
\hline Preoperative beta-blockade & 45 & $8(62 \%)$ & $14(44 \%)$ & 0.337 \\
\hline Laparoscopic approach* & 44 & $2 / 12(17 \%)$ & $16(50 \%)$ & 0.083 \\
\hline Epidural analgesia & 45 & $9(69 \%)$ & $12(38 \%)$ & 0.098 \\
\hline Intraoperative fluid volume (L) & 41 & $2.5(2.0-4.0)$ & $3.0(1.5-4.0)$ & 0.972 \\
\hline Postoperative fluid volume ( $<24$ hours, L) & 42 & $3.4(2.6-3.7)$ & $1.8(1.2-3.1)$ & $0.003^{\#}$ \\
\hline \multicolumn{5}{|l|}{ Outcomes } \\
\hline Intraoperative hypertension & 42 & $5(39 \%)$ & $20 / 29(69 \%)$ & 0.092 \\
\hline Postoperative complications & 45 & $3(23 \%)$ & $6(19 \%)$ & 0.704 \\
\hline
\end{tabular}

Binary factors are reported as $\mathrm{n}(\%)$ or as $\mathrm{n} / \mathrm{N}(\%)$ where there are missing data, with $\mathrm{P}$ values from Fisher's exact tests. Continuous factors are reported as mean $\pm \mathrm{SD}$, with $\mathrm{P}$ values from $t$-tests, or as median (IQR), with $\mathrm{P}$ values from Mann-Whitney tests. ", $\mathrm{P}<0.05$; *, excludes one patient whose laparoscopic procedure was converted to open surgery.

first 24 hours postoperatively was found to be significantly higher in those that developed postoperative hypotension (median: 3.1 vs. $1.6 \mathrm{~L}, \mathrm{P}=0.020$ ), with a similar difference observed for those undergoing postoperative vasopressor therapy (median: 3.4 vs. $1.8 \mathrm{~L}, \mathrm{P}=0.003$ ).

\section{Postoperative outcomes}

A total of 9 patients (20\%) developed postoperative complications, including 8 patients after $\mathrm{OA}$ and 1 patient after LA ( $31 \%$ vs. $5 \%$; Fisher's exact test $\mathrm{P}=0.058)$. Complications included pneumonia $(\mathrm{N}=3)$, acute kidney injury $(\mathrm{N}=1)$, wound infection $(\mathrm{N}=1)$, pancreatic fistula $(\mathrm{N}=1)$, haematoma $(\mathrm{N}=2)$ and cardiogenic shock $(\mathrm{N}=1)$. There were two severe complications after open surgery: one patient developed respiratory failure secondary to pneumonia and one patient developed cardiogenic shock intraoperatively and died 2 days later from multi-organ failure. Complication rates were significant lower in patients with intraoperative hypertension compared to those without $(8 \%$ vs. $41 \% ; \mathrm{P}=0.019)$, but there was no significant association with postoperative hypotension $(\mathrm{P}=0.469)$ or vasopressor therapy $(\mathrm{P}=0.704)$.

The median postoperative length of stay in intensive care and hospital stays were 1 (range, 1-7) and 5 (range, 1-15) days, respectively. The median length of hospital stay was significantly shorter after LA compared to OA (median: 3 vs. 5 days; $\mathrm{P}<0.001)$. Lower plasma normetanephrine levels $(\mathrm{P}=0.006)$ and the use of epidural analgesia $(\mathrm{P}=0.004)$ were also associated with significantly longer lengths of stay (Table 5). On multivariable analysis, OA was the only factor found to be a significant independent predictor of 
Table 5 Factors associated with length of stay

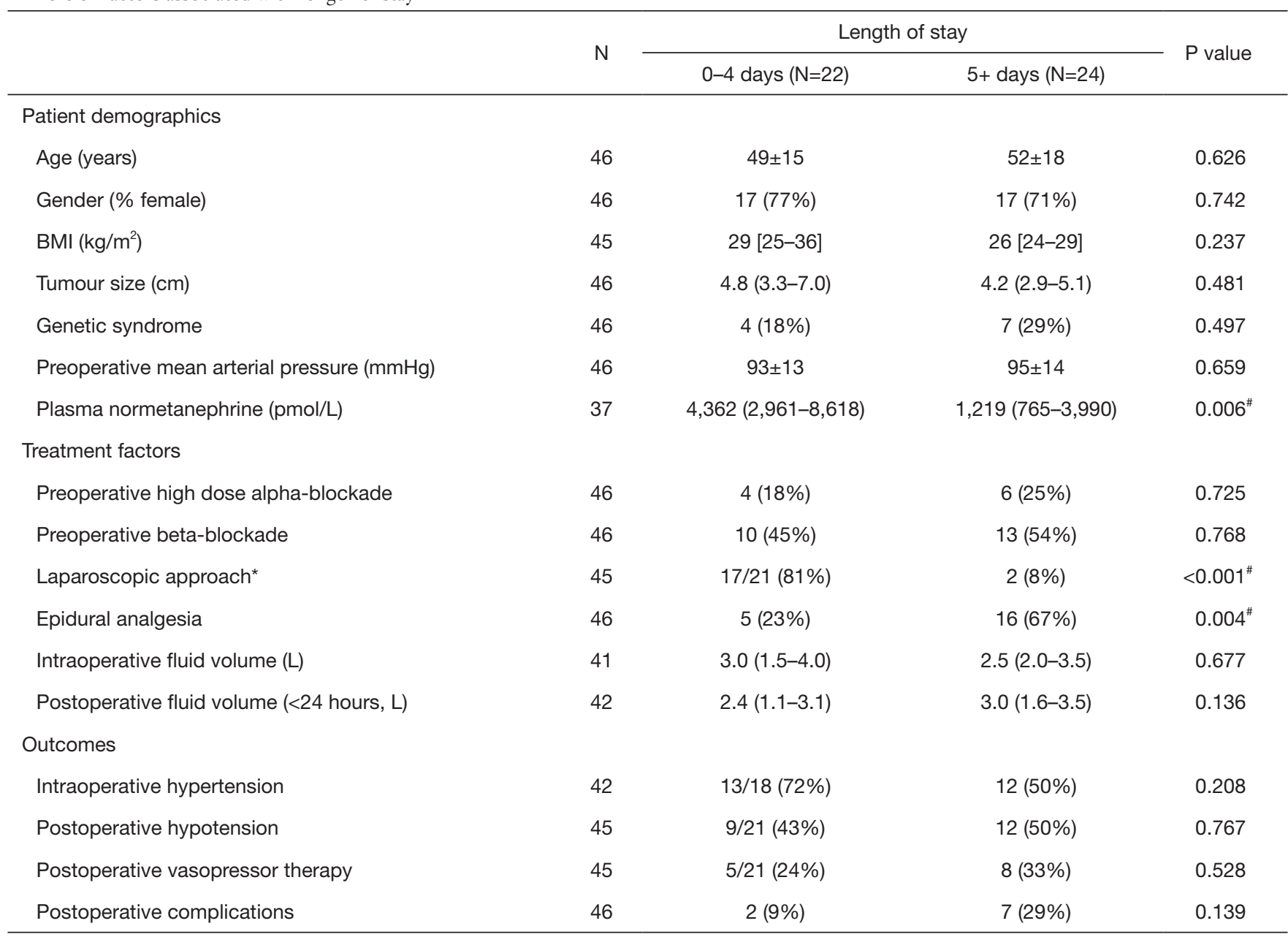

Binary factors are reported as $\mathrm{n}(\%)$ or as $\mathrm{n} / \mathrm{N}(\%)$ where there are missing data, with $\mathrm{P}$ values from Fisher's exact tests. Continuous factors are reported as mean $\pm \mathrm{SD}$, with $\mathrm{P}$ values from $t$-tests, or as median (IQR), with $\mathrm{P}$ values from Mann-Whitney tests. " $\mathrm{P}<0.05$; *, excludes one patient whose laparoscopic procedure was converted to open surgery.

prolonged length of hospital stay ( $>5$ days), with an odds ratio of 42 (95\% CI: 6-288, $\mathrm{P}<0.001)$, relative to $\mathrm{LA}$.

\section{Discussion}

This study has evaluated the incidence, risk factors and clinical significance of perioperative HDI in a contemporary series of patients undergoing adrenalectomy for phaeochromocytoma. An important finding is that patients with hormonally active tumours, as suggested by very high plasma normetanephrine levels, are at particular risk of intraoperative hypertension and this is consistent with findings from other studies $(16,19,20)$. Intraoperative hypertension was common, occurring in $60 \%$ of patients, but interestingly was associated with a lower complication rate. This is an unexpected finding, and is contrary to a study by Brunaud et al., who identified intraoperative HDI as an independent predictor of all-cause and cardiovascular morbidity after LA for phaeochromocytoma (14). The reason for this discrepancy is unclear, but may be due to the fact that complication rates were lower in the LA group (although not significantly so), and patients in this group were more prone to intraoperative hypertension, due to the presence of larger, more metabolically active tumours than in the OA group. Whilst we attempted to account for the effect of confounding factors such as these using multivariable analyses, the small sample size and relatively low complication rate will have resulted in insufficient 
statistical power to detect all but the strongest of associations. As a result, these analyses are unlikely to have accounted for all of the confounding effects and selection biases present in the data. Perioperative cardiovascular morbidity occurred in only one patient in our study, and this may be a reflection of rapid control of intraoperative HDI by an experienced anaesthetic team.

Morbidity and mortality after surgery for phaeochromocytoma have reduced significantly over recent decades, due to improvements in preoperative management and prompt intraoperative control of HDI (21). In a recent study by Livingstone et al. (21), there was a significant association between preoperative phenoxybenzamine dose and intraoperative haemodynamic stability. Doxazosin has been the preferred alpha-blocker in our Unit since 2014, although a high dose did not significantly influence either intraoperative or postoperative haemodynamics in our study.

Early studies suggested that LA may increase the risk of intraoperative HDI due to the effect of the pneumoperitoneum, although data is limited and conflicting (20,22-24). There was a significant association between LA and intraoperative hypertension in our study, although this finding may be due to a preponderance of hormonally active tumours in the LA group, as suggested by higher plasma normetanephrine levels (Table 1), and LA was not found to be an independent predictor of intraoperative hypertension on multivariable analysis.

After surgical resection of a phaeochromocytoma, patients are at risk of postoperative hypotension. Almost half of the patients in our study had postoperative hypotension, including $29 \%$ who required postoperative vasopressor therapy. Preoperative beta-blockade therapy was the only independent predictor of postoperative hypotension in our study. Although postoperative hypotension is well recognised after adrenalectomy for phaeochromocytoma $(25,26)$, few studies have evaluated the risk factors for its occurrence. In a study of 41 patients, Benay et al. identified preoperative mean arterial pressure, catecholamine levels and resection of another organ as risk factors for postoperative HDI (17). In a Japanese study, catecholamine levels and tumour size were also found to be significant predictors of postoperative vasopressor therapy (26). Plasma normetanephrine levels and tumour size were higher in patients with postoperative hypotension in our study, although the difference was only significant for the latter (Table 3). Postoperative hypotension is probably due to a combination of the persistence of circulating antihypertensive drugs, reversal of chronic vasospasm after tumour resection, and/or down-regulation of adrenergic receptors $(20,26,27)$. Although postoperative hypotension was transient and did not adversely impact on clinical outcomes in our study, it was associated with significantly more intravenous fluid administered in the first 24 hours postoperatively (Table 3). Due to the potential need for vasopressor therapy, recently published guidelines from the British Association of Endocrine and Thyroid Surgeons (BAETS) have recommended that all patients undergoing adrenalectomy for phaeochromocytoma should be monitored in an intensive care unit (15). However, intensive care is a limited and expensive resource and our data suggests that less than one third of patients require postoperative vasopressors. In the study by Benay et al., selective use of intensive monitoring has been recommended in low risk patients after a 6-hour period of stability (17). A recent French study by Lentschener et al. found that more than half of patients fulfilled criteria for transfer to a ward after three hours of postoperative monitoring in recovery (28). In our study, no patients with small tumours $<5 \mathrm{~cm}$ required postoperative vasopressor support after laparoscopic surgery compared to $39 \%$ after open surgery $(\mathrm{P}=0.059)$, and this is likely to reflect the lower rate of epidural use since our laparoscopic programme began (11\% vs. $73 \% ; \mathrm{P}<0.001)$.

This study was subject to two main limitations, with the primary one being the relatively small sample size. As a result of this, the statistical power of the analyses will have been limited, meaning that only large differences between groups could be detected. Consequently, the false-negative rate will have been inflated, meaning that true effects may have been missed. With regards to the multivariable analysis, the combination of the small sample size and low rate of some outcomes meant that it was only feasible to analyse the data using a stepwise approach, in order to minimise the number of factors in the model and prevent over-fitting. This resulted in very simple models, which only selected the single strongest predictors of outcome for inclusion, and so will not have fully accounted for any selection bias or confounding effects present in the data. The results of the multivariable analyses must be interpreted in the light of this, and would ideally require validation in a larger external cohort to confirm the findings. The second limitation was the fact that data were not complete for all factors, with plasma normetanephrine levels being unavailable for $20 \%$ of the cohort, and intraoperative haemodynamic data missing for $9 \%$. Excluding cases with missing data from the analysis of the respective factors 
further reduced the sample size, further escalating the aforementioned issues with statistical power. In addition, excluding these cases may have introduced a degree of selection bias if the data were not missing at random.

In conclusion, intraoperative hypertension is a common event during adrenalectomy for phaeochromocytoma, and is more likely in patients with large tumours and very high preoperative plasma normetanephrine levels. Cardiovascular complications may be prevented by prompt treatment of intraoperative HDI by an experienced anaesthetic team. The risk of postoperative hypotension may be reduced by limiting the use of preoperative beta-blockade therapy and routine intensive care monitoring may not be necessary in low risk patients.

\section{Acknowledgments}

None.

\section{Footnote}

Conflicts of Interest: The authors have no conflicts of interest to declare.

Ethical Statement: The authors are accountable for all aspects of the work in ensuring that questions related to the accuracy or integrity of any part of the work are appropriately investigated and resolved. The study was registered and approved as a clinical audit by University of Birmingham NHS Foundation Trust (Audit number CARMS-13194). Informed consent was waived in accordance with the ethical committee opinion.

\section{References}

1. Pacak K, Linehan WM, Eisenhofer G, et al. Recent advances in genetics, diagnosis, localization, and treatment of pheochromocytoma. Ann Intern Med 2001;134:315-29.

2. Lenders JW, Eisenhofer G, Mannelli M, et al. Phaeochromocytoma. Lancet 2005;366:665-75.

3. Connor D, Boumphrey S. Perioperative care of phaeochromocytoma. BJA Education 2016;16:153-8.

4. Lentschener C, Gaujoux S, Tesniere A, et al. Point of controversy: perioperative care of patients undergoing pheochromocytoma removal-time for a reappraisal? Eur J Endocrinol 2011;165:365-73.

5. Ross EJ, Prichard BN, Kaufman L, et al. Preoperative and operative management of patients with phaeochromocytoma. Br Med J 1967;1:191-8.

6. Thompson JE, Arrowood JG. Pheochromocytoma; surgical and anesthetic management. Anesthesiology 1954;15:658-65.

7. Desmonts JM, le Houelleur J, Remond P, et al. Anaesthetic management of patients with phaeochromocytoma. A review of 102 cases. Br J Anaesth 1977;49:991-8.

8. Hull CJ. Phaeochromocytoma. Diagnosis, preoperative preparation and anaesthetic management. Br J Anaesth 1986;58:1453-68.

9. Kinney MA, Warner ME, vanHeerden JA, et al. Perianesthetic risks and outcomes of pheochromocytoma and paraganglioma resection. Anesth Analg 2000;91:1118-23.

10. van Heerden JA, Roland CF, Carney JA, et al. Longterm evaluation following resection of apparently benign pheochromocytoma(s)/paraganglioma(s). World J Surg 1990;14:325-9.

11. Pisarska M, Pędziwiatr M, Budzyński A. Perioperative hemodynamic instability in patients undergoing laparoscopic adrenalectomy for pheochromocytoma. Gland Surg 2016;5:506-11.

12. Joris JL, Hamoir EE, Hartstein GM, et al. Hemodynamic changes and catecholamine release during laparoscopic adrenalectomy for pheochromocytoma. Anesth Analg 1999;88:16-21.

13. Prys-Roberts C, Farndon JR. Efficacy and safety of doxazosin for perioperative management of patients with pheochromocytoma. World J Surg 2002;26:1037-42.

14. Brunaud L, Nguyen-Thi PL, Mirallie E, et al. Predictive factors for postoperative morbidity after laparoscopic adrenalectomy for pheochromocytoma: a multicenter retrospective analysis in 225 patients. Surg Endosc 2016;30:1051-9.

15. BAETS. Adrenal Surgery Practice Guidance for the UK. Secondary Adrenal Surgery Practice Guidance for the UK 2016. Available online: http://www.baets.org.uk/wpcontent/uploads/Adrenal-Surgery-Practice-Guidance-forthe-UK-2016.pdf

16. Gaujoux S, Bonnet S, Lentschener C, et al. Preoperative risk factors of hemodynamic instability during laparoscopic adrenalectomy for pheochromocytoma. Surg Endosc 2016;30:2984-93.

17. Bénay CE, Tahiri M, Lee L, et al. Selective strategy for intensive monitoring after pheochromocytoma resection. Surgery 2016;159:275-82.

18. Dindo D, Demartines N, Clavien PA. Classification of surgical complications: a new proposal with evaluation in 
a cohort of 6336 patients and results of a survey. Ann Surg 2004;240:205-13.

19. Bruynzeel H, Feelders RA, Groenland TH, et al. Risk Factors for Hemodynamic Instability during Surgery for Pheochromocytoma. J Clin Endocrinol Metab 2010;95:678-85

20. Kiernan CM, Du L, Chen X, et al. Predictors of hemodynamic instability during surgery for pheochromocytoma. Ann Surg Oncol 2014;21:3865-71.

21. Livingstone M, Duttchen K, Thompson J, et al. Hemodynamic Stability During Pheochromocytoma Resection: Lessons Learned Over the Last Two Decades. Ann Surg Oncol 2015;22:4175-80.

22. Davies MJ, McGlade DP, Banting SW. A comparison of open and laparoscopic approaches to adrenalectomy in patients with phaeochromocytoma. Anaesth Intensive Care 2004;32:224-9.

23. Inabnet $\mathrm{WB}$, Pitre J, Bernard D, et al. Comparison of the hemodynamic parameters of open and laparoscopic adrenalectomy for pheochromocytoma. World J Surg

Cite this article as: Thompson JP, Bennett D, Hodson J, Asia M, Ayuk J, O’Reilly MW, Karavitaki N, Arlt W, Sutcliffe RP. Incidence, risk factors and clinical significance of postoperative haemodynamic instability after adrenalectomy for phaeochromocytoma. Gland Surg 2019;8(6):729-739. doi: 10.21037 /gs.2019.11.22
2000;24:574-8.

24. Sprung J, O'Hara JF Jr, Gill IS, et al. Anesthetic aspects of laparoscopic and open adrenalectomy for pheochromocytoma. Urology 2000;55:339-43.

25. Kasahara T, Nishiyama T, Takahashi K. Laparoscopic adrenalectomy for pheochromocytoma: evaluation of experience and strategy at a single institute. BJU Int 2009;103:218-22.

26. Namekawa T, Utsumi T, Kawamura K, et al. Clinical predictors of prolonged postresection hypotension after laparoscopic adrenalectomy for pheochromocytoma. Surgery 2016;159:763-70.

27. Streeten DH, Anderson GH Jr. Mechanisms of orthostatic hypotension and tachycardia in patients with pheochromocytoma. Am J Hypertens 1996;9:760-9.

28. Lentschener C, Gaujoux S, Mion G, et al. Most patients undergoing phaeochromocytoma removal could be safely discharged from the post-anaesthesia care unit to the ward after three hours monitoring. Br J Anaesth 2018;120:879-80. 